\title{
Does acquiring verbal reference enable learning from verbal testimony?
}

\section{Research Highlights}

- This investigation asks how, and how early, we begin to use linguistic information alone to learn about new objects and events to which we have no perceptual access.

- Adopting a longitudinal approach, we assess the relation between children's verbal reference capacity at 15 months and their facility in learning from verbal testimony at 24 months.

- We seek to specify this relation between verbal reference and verbal testimony, controlling for more general advances in memory and attention between 15 and 24 months.

- This investigation offers new insights into how children's emerging language, cognitive, and social-communicative capacities support knowledge acquisition. 


\title{
Does acquiring verbal reference enable learning from verbal testimony?
}

\begin{abstract}
The human ability to learn from verbal testimony - information offered by others about objects, events, or ideas - increases exponentially our communicative and representational reach, permitting us to learn, communicate, and reason about entities for which we have no direct perceptual experience. But how does this ability develop? We propose that this fundamentally human capacity rests upon the acquisition of 'verbal reference' - a referential link between words, their referents, and mental representations of such referents. If this is the case, then children who acquire verbal reference early will begin to learn from testimony earlier, and this should have downstream consequences for learning. To test this hypothesis, we adopt a longitudinal design, asking whether children's command of verbal reference at 15 months predicts their ability to learn from verbal testimony alone at 24 months. By controlling for advances in memory, attentional control, and vocabulary over this developmental period, we assess whether this relation is tied specifically to verbal reference. We discuss the implications for subsequent language and cognitive development.
\end{abstract} Keywords: verbal reference, verbal testimony, mental representation, longitudinal design 


\section{Does acquiring verbal reference enable learning from verbal testimony?}

In the course of our daily lives, we learn considerably about objects, events and ideas to which we have no direct perceptual access (Deacon, 1997; Miller, 1990). Imagine, for example, listening to someone discuss an upcoming Winter Olympics, an event from the remote past, or about concepts as abstract as 'singularity'. In each of these examples, we learn from others' verbal testimony - that is, others' use of language that permits us to gain new information about which we have no direct perceptual access, and to create new mental representations of objects, events, or ideas. Verbal testimony allows us to transmit information across space and time, imagine fictional characters and mythical worlds, and even form beliefs about the supernatural (see Harris \& Lane, 2014). Learning from verbal testimony is thus crucial for cognitive development. Children as young as 18 months are able to learn new words and new facts from speech alone (Akhtar, 2005; Floor \& Akhtar, 2006; Foushee et al., 2021), including verbal testimony (Anderson \& Pempek, 2005; O’Doherty et al., 2011; Robb et al., 2009). At roughly the same age, children use the quality of others' verbal testimony to make inferences about their knowledge and social characteristics (e.g., Brooker \& Poulin-Dubois, 2013; Harris \& Corriveau, 2011; Harris \& Lane, 2014; Koenig \& Harris, 2005; Luchkina et al., 2018; Sobel \& Kushnir, 2013).

Inquiries into the relation between language and learning have long been a focus in philosophy, psychology and linguistics - from Plato's efforts in his dialogues to connect objects, words, knowledge and knowers (see Scolnikov, 2006, for a review) to Locke's claims that words excite one's internal conceptions of objects and are used under the assumption that others have similar internal conceptions excited by the same words (see Locke, 1847; first publised in 1689). The long history of these ideas is a testament to their significance: how and when do we develop 
the ability to use language to transmit information from one mind to another? We propose that to learn from testimony, children must first have acquired verbal reference - a referential link between words, their referents, and mental representations of such referents (Bloom, 1993; Clark \& Bangerter, 2004; Waxman \& Gelman, 2009; Waxman \& Lidz, 2007; Luchkina \& Waxman, 2021a; Luchkina \& Xu, 2021).

In recent years, researchers have made significant progress in identifying the developmental timeline of both verbal reference and verbal testimony. For example, verbal reference appears to emerge at around 12 months ${ }^{1}$, but that it is initially fragile. This fragility is evident in tasks in which children hear the familiar name of an absent referent (e.g., a dog) and are then asked to locate it. Twelve-month-olds rely on perceptual 'anchors' - visible placeholders that mark the location of the now-absent object (e.g., a box; see Gallerani et al., 2009; Ganea \& Saylor, 2013; Osina et al., 2013, 2014; Saylor, 2004). Without such reminders, 12-month-olds fail to retrieve a representation of a now-absent object (e.g., Luchkina et al., 2020). By 14-16 months, however, such anchors are no longer required: children successfully retrieve representations of familiar absent objects upon hearing their names (Hendrickson \& Sundara, 2017; Luchkina et al., 2020); they also successfully modify these representations on the basis of verbal information alone (Ganea et al., 2016). The most recent evidence shows that 15month-olds successfully establish a representation of an absent novel object and map it to a novel word (Luchkina \& Waxman, 2022). Together, these findings suggest that by 15 months, children have acquired verbal reference: they (1) successfully link words and mental representations and (2) know that words can communicate about objects or events, even when these are perceptually unavailable. 
Extant evidence also suggests that by 22 months, children successfully learn new information from verbal testimony. That is, they learn new information that has been communicated by language alone, with no perceptual access to objects, events, or ideas described. (Galazka \& Ganea, 2014; Ganea et al., 2007). In Ganea et al. (2007), for example, children were asked to identify the toy, one they had seen before, after being told that water had spilled all over it and it had become wet. By 22 (but not 19) months, children successfully updated their mental representation of that toy based on verbal testimony, correctly choosing a wet toy over an identical-looking dry one ${ }^{2}$. At 30 months, this capacity deepens: children now use verbal testimony to establish mental representations of novel objects (e.g., "Grimps live in the ocean ... Grimps are gray"; see Saylor et al., 2016) and novel events (e.g., Arunachalam \& Waxman, 2010) that they had never seen; they can also modify their representation of a hidden object's location (“E2 put the puppy behind the pillow!”; see Ganea \& Harris, 2010).

In sum, this developmental timeline is consistent with our proposal that verbal reference is a pre-requisite for learning from verbal testimony. However, there is a gap: There is no evidence documenting a predictive relation between children's acquisition of verbal reference and their ability to learn from verbal testimony. In the current paper, we fill this gap by testing three specific hypotheses.

First, we hypothesize that variability in infants' command of verbal reference at 15 months predicts variability in their ability to learn from verbal testimony at 24 months. Testing infants at 15 months (Time 1) permits us to assess verbal reference in infants when they first succeed, as a group, to link a novel word to a representation of an absent novel object. It also provides an excellent entry point to the longitudinal analysis because there is substantial variability in individual performance in this task at 15 months (Luchkina \& Waxman, 2022). Our 
decision to select 24 months (Time 2) is also well-motivated. At 24 months, children succeed, as a group, in learning about objects from verbal testimony alone, but here too, there is substantial variability in individual performance (e.g., Galazka \& Ganea, 2014; Ganea \& Saylor, 2013). Indeed, pilot data from our lab (see below) provides strong assurances that we will capture this in our proposed verbal testimony task at 24 months.

Second, we hypothesize that differences in participants' command of verbal reference may persist into 24 months and may also have concurrent effects on learning from testimony. Indeed, there is reason to suspect that both longitudinal and concurrent relations between verbal reference and verbal testimony may be mediated by children's vocabulary at 24 months. For example, it is possible that the development of verbal reference promotes vocabulary growth, which, in turn, promotes learning from verbal testimony. We propose a planned mediation analysis to explore the relations between these measures.

Third, we hypothesize that both longitudinal and concurrent effects of verbal reference on learning from verbal testimony may be distinct from more general developmental advances in memory and attention. To test this prediction, we present infants with two non-linguistic tasks at both 15 and 24 months. The visual short-term memory task (VSTM) provides an index of infant memory capacity (Cheng et al., 2019; Ross-Sheehy, 2003; see Astle \& Scerif, 2011, for a review); the Spatial Cueing task measures visual attentional control (see Arredondo et al., 2022). If, as we predict, the relation between verbal reference and verbal testimony is specific to language, then this relation should persist even when controlling for infant visual memory and attentional control.

\section{Method}




\section{Power analysis}

We reasoned that compelling evidence would require at least a medium effect size of condition (Cohen, 2013) in each task. For the verbal reference task, a power analysis using linear multiple regression with random effects $\left(\mathrm{G}^{*}\right.$ Power 3.1; Faul et al., 2007) revealed a required $N=68$ with a target effect size $f^{2} \geq .1225$ (medium), alpha=.05, power $=.8, \mathrm{H}_{1} \rho^{2}=0.2, \mathrm{H}_{0} \rho^{2}=0$, number of predictors $=.1$ For the verbal testimony task, a power analysis using a model with the same parameters revealed a required sample of $N=82$. For the spatial cueing task, the sample size estimate is based on Arredondo et al. (2022) who tested 60 children per age group. For the VSTM task, the sample size estimate is based on Ross-Sheehy et al. (2003) who tested 48 children per age group.

In sum, our power analyses for each task show that $N=82$ at Time 2 (24 months) will satisfy the requirements of each task. To accommodate the possibility of low test-retest reliability of infant and toddler looking behavior, we increase the $n$ by $50 \%$, resulting in a sample size of $N=123$. To accommodate an anticipated longitudinal attrition rate of roughly $28 \%$ (based on prior longitudinal studies at our lab), we oversample at Time 1 (15 months), recruiting 170 participants to ensure a yield of at least 122 participants at 24 months. Thus, total sample recruited at 15 months is $N=170$, with an anticipated $N=122$ at 24 months.

\section{Participants}

One hundred and seventy ( $N=170)$ US-based English-acquiring 15-month-old children with no known developmental disabilities will be recruited. We will recruit an approximately equal number of male and female children, sampling broadly so that the composition of our sample reflects the racial and ethnic diversity of the local community (if tested in-lab) or the US 
(if tested online). After each visit to the lab (or online participation), parents or legal guardians will receive either $\$ 5$ or a small toy or a baby t-shirt with our lab’s logo.

\section{Surveys}

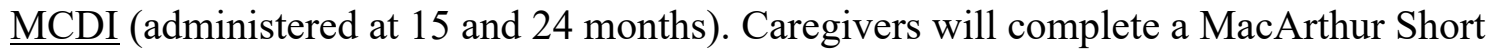
Form Vocabulary Checklist: Level II (MCDI II, Fenson et al., 2000), augmented with any familiar words introduced in the experiments that are not already included.

Demographic survey (administered at 15 and 24 months). This will identify caregivers' education, employment, gender, race, and children's exposure to non-native languages.

\section{Experimental tasks}

At the 15-month visit, children will participate in the verbal reference, VSTM and Spatial Cueing tasks. At the 24-month visit, children will participate in the verbal reference, verbal testimony, VSTM, and Spatial Cueing tasks. We expect all children to complete all tasks: evidence from our lab and others indicates that 15- and 24-month-olds can maintain focus for the 10 minutes required to administer all tasks. Nevertheless, to ensure that we have sufficient power for the verbal tasks, we present these tasks first, followed by the non-verbal tasks presented in a random order.

\section{Verbal reference task: administered at 15 and 24 months}

\section{Stimuli}

Stimuli and procedure will be identical to that of the Semantic Priming condition ${ }^{3}$ in Luchkina and Waxman (2021b; see Figure 1a). On each trial, children will view a video-taped vignette including a Priming phase and a Test phase, presented on a computer screen. The names of familiar objects - a cat, a dog, a horse, a hat, a sock, a jacket, a car, a bus, a truck, an apple, an orange, and a banana - presented during Priming are known, on average, by at least $60 \%$ of 15 - 
month-olds (according to Wordbank data; see Frank et al., 2017). Novel objects presented during Test are less familiar to 15-month-olds objects whose names they do not know: one from the same semantic neighborhood as the Priming phase and the other from a different neighborhood (see Figure 1b). When children participate in this task again at 24 months, we introduce a different set of novel words to minimize the unlikely possibility that children retained the wordreferent mappings established at 15 months over the intervening 9-month period.

\section{Procedure}

All infants will participate in four trials, each including a Priming phase and a Test phase (Figure 1a). Each trial will include one novel object-novel word pairing. During the Priming phase, an actor first points to and names three familiar objects from the same semantic neighborhood (e.g., an apple, an orange, and a banana). The objects appear one at a time, behind the actor; the actor turns to point to and name each (e.g., "Look! An apple! Do you see the apple?"). Next the actor looks toward an object hidden behind her back (seemly accidentally), and names it with a novel noun (e.g., "Look, a modi! That's a nice modi! I like modis!'), looking alternately toward the occluded object and the child. The object remans hidden throughout. During the Test phase, two objects - one from the same semantic neighborhood as the familiar objects (e.g., a novel fruit) demonstrated during Priming and one from a semantically distant neighborhood (e.g., a furniture item) - appear side-by-side. Children are prompted with the novel word that was taught during the Priming phase - e.g., "Where is the modi". The images appear on display immediately after the target word is uttered. After a $2 \mathrm{~s}$ delay, they hear a second prompt, e.g., "Can you find the modi?". Test images remain on display for $6 \mathrm{~s}$ in total.

\section{Looking time data preparation}


We will analyze children's looking behavior from the onset of the image presentation through the end of the trial ( $6 \mathrm{~s}$; see Figure 1). For data collected in the lab using a Tobii T60XL eyetracker, we identify areas of interest (AOI) of approximately $720 * 790$ pixels around each of the object images, such that every AOI includes a 50-pixel-wide area around an object image. This 50-pixel margin is added to accommodate for noise in eyetracking data, which often results from children's head movement or blinking. Gazes outside these areas will be excluded from analysis (and marked as 'away'), as will be trials in which total looking time is less than $1 \mathrm{~s}$ (see, e.g., Ferguson et al., 2018).

For data collected on Lookit, manual frame-by-frame coding will be performed by trained research assistants, using Datavyu (https://datavyu.com). Datavyu is a free open-source software application that records the frequencies and durations of specific behaviors. Research assistants will watch video-recordings of online testing sessions and determine whether the child is looking to the left or to the right side of the screen during each frame. Depending on the location of the target object, left-right codes will then be converted to target-distractor codes by a different research assistant. Ten percent of all videos will be selected for reliability coding and coded by a different research assistant. Disagreements between the coders will be resolved by a third independent coder.

All research assistants involved in video coding will be agnostic to experimental conditions. They will only see the direction of participants' gaze and will not see visual stimuli or hear audio stimuli that were presented during the experimental session. These measures insure that during frame-by-frame coding, research assistants cannot infer the side of the target object from infants' looking behavior and do not introduce a systematic bias into their coding. 


\section{Dependent variables}

For each trial of each child, we will calculate the net ${ }^{4}$ proportion of looking time devoted to the target throughout the analysis window.

The net looking preference for the target object $\left(\mathrm{LT}_{\mathrm{vr}}^{5}\right)$ equals the proportion of looking time to the target object after the verbal prompt onset (at $2000 \mathrm{~ms}$ ) minus the proportion of looking time to the target object before the verbal prompt:

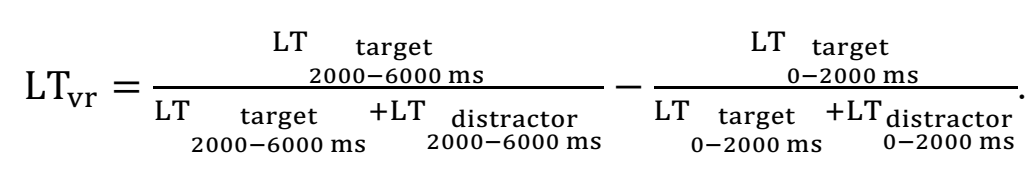

To assess the time course of children's attention to target images, eyetracker data will be aggregated by condition (when applicable) into a series of 100-ms bins. For data collected on Lookit, we will use 200-ms bins (families' web-cameras typically have sampling rates varying between $30 \mathrm{HZ}$ and $60 \mathrm{HZ}$, which translates to frame duration ranging from $16.67 \mathrm{~ms}$ to $33.33 \mathrm{~ms}$; binning our data into 200-ms segments allows us to have at least 6 frames in each bin and to calculate the proportion of children's looking to the target image).

\section{Preliminary analyses}

At each age, preliminary analyses will test the effects of child vocabulary (MCDI II), demographic factors, language exposure, children's attention to the screen during Priming, and trial order Analyses will be implemented by fitting Generalized Linear Models (GLM) using the glm function of the Stats package in R (R Core Team, 2013). If any such factor is significant in preliminary analyses, we will include it in main analyses.

\section{Analyses and Predictions}

In analyzing children's performance on the verbal reference task at each age, we will fit a GLM to net looking preference for the target object ( $\mathrm{LT}_{\mathrm{vr}}$ ) using the glm function in the Stats 
package in $\mathrm{R}$ to evaluate the effects of Test Item. We test the effects of Test Item because there can be differences in the knowledge domains.

We expect that overall, children will prefer looking at the target test object. Specifically, we expect children's preference for the target will be greater in response to the test question than during baseline preference assessed during the first $2 \mathrm{~s}$ of silent presentation (which will manifest as a significant effect of the intercept in our model). We anticipate no effect of Test Item. However, if such an effect is observed, we will conduct follow-up contrasts for each test trial to assess whether these effects reflect differences in lexical knowledge (e.g., whether children perform worse on test items where they knew fewer object names used during priming).

To examine the relation between children's performance on the verbal reference task at 15 and 24 months, we will fit a GLM with $\mathrm{LT}_{\mathrm{vr}}$ at 15 months as a predictor for $\mathrm{LT}_{\mathrm{vr}}$ at 24 months. This will allow us to evaluate developmental stability within participants over time (i.e., whether children's relative performance on the verbal reference task persists over time). We will fit another GLM to the combined data from both experiments with age (15 vs. 24 months), as a predictor to assess developmental change within participants.

We predict two sets of effects based on these analyses. (1) Developmental stability: If differences in children's performance on the verbal reference task persist over time (because of individual differences in the development of verbal refence), the effect of task performance at 15 months on task performance at 24 months will be significant. (2) Developmental change: We predict that children will improve with age, as indicated by a significant effect of age.

\section{Results and Discussion}

To be added after data collection and analyses are complete. 


\section{Verbal testimony task: administered at 24 months}

\section{Stimuli}

On each trial, the experimenter names a novel creature and describes its typical habitat and color (see Figure 2a). These verbal stimuli were chosen according to children's comprehension skills: by 24 months, children understand several color words (Forbes \& Plunkett, 2019; Frank et al., 2017). Each child will be randomly assigned either to the Experimental or to the Control condition (between-subjects) and complete four trials. Test stimuli for each trial are novel-looking creatures of basic colors (in English) depicted in their habitat (see Figure 2b).

\section{Procedure}

Experimental condition $(N=61)$. During Introduction (Figure 2a), children see an actor on a video-recording on a computer screen, shown against a neutral background. She describes and names a novel creature, giving its typical habitat and color, with no referent present. The creature's name, habitat, and color are mentioned twice (e.g., "Hi, baby! Did you know? Grimps are green and they live in water. Did you know that? Grimps are green and they live in water!").

During Test (Figure 2a), children see two objects - a target and a distractor; they are asked to identify the target by looking to one of the objects. The target object matches the description presented during the introduction in both color and habitat. The distractor shares no features with the description (neither color nor habitat). Right before image presentation, children hear the novel word, e.g., "Where is the grimp?". Immediately after, images appear on the screen. After a 2 s delay, they hear a verbal prompt, e.g., "Can you find the grimp?". Test images remain on display for $6 \mathrm{~s}$ in total. 
Control condition $(N=61)$. The Control condition is identical to the Experimental condition, except during the Introduction phase: the experimenter describes and names a novel creature, but does not provide information about it's color or habitat. The Test phase is identical to the Experimental condition. This condition allows us to evaluate whether children have any strong intrinsic preferences that are not accounted for by subtracting the looking preference during the silent image presentation for either image presented during Test.

\section{Looking time data preparation}

Identical to the verbal refence task.

\section{Dependent variables}

The main dependent variable is the looking preference for the target ${ }^{6}$ object $\left(\mathrm{LT}_{\mathrm{vt}}\right)$, calculated as the proportion of looking time to the target object of the total looking time to either the target or the distractor object:

$$
\mathrm{LT}_{\mathrm{vt}}=\frac{\mathrm{LT}_{\text {target }}}{\mathrm{LT}_{\text {target }}+\mathrm{LT}_{\text {distractor }}} .
$$

In addition to analyzing the overall looking preference for the target object, we will examine the timecourse of children's looking preference from the onset of the verbal prompt (2000 ms) until the end of the trial $(6000 \mathrm{~ms})$. We are interested to learn about the timing of divergences in children's looking behavior between the conditions.

\section{Preliminary analyses}

Preliminary analyses will test the effects of child vocabulary (MCDI II), demographic factors, language exposure, children's attention to the screen during Introduction, and trial order Analyses will be implemented by fitting Generalized Linear Models (GLM) using the glm function of the Stats package in R (R Core Team, 2013). If any such factor is significant in preliminary analyses, we will include it in main analyses. 


\section{Analyses and Predictions}

In analyzing children's performance on the verbal testimony task, we will first fit a GLM to test the effect of Condition on the overall net looking preference for the target $\left(\mathrm{LT}_{\mathrm{vt}}\right)$. We predict a significant effect of Condition. Children in the Experimental condition should show significantly stronger preferences for the target test object than children in the Control condition. Only children in the Experimental condition should show a preference for the target test object.

In analyzing the time course data, we will use the cluster-based permutation analysis using the eyetrackingR package in R (Dink \& Ferguson, 2015) to identify significant divergences between the conditions. We expect a significant divergence between the conditions starting from the onset of the target word (see Luchkina \& Waxman, 2021b).

\section{Pilot Data}

We have completed a pilot study with 15 children tested in the Experimental condition to ensure that 24-month-olds succeed on the task as a group and that there is enough spread in their performance to support the analysis of individual differences. All children $\left(M_{\mathrm{age}}=24.6\right.$ months, 8 girls) were recruited and tested on Lookit. Data from two children were excluded because children's eyes were not clearly visible during the recording. Data from 13 children were analyzed. We excluded trials on which children looked away from the screen more than $30 \%$ at Test. The total number of analyzed trials was 40 . Our analyses indicate that children's looking preference was significantly above chance $(50 \%), M_{\mathrm{LTvt}}=60.2 \%, S D=20.6 \%, t=3.1, p=.002$ (see Figure 2c). This pilot test provides strong assurances that the verbal testimony task is wellsuited to test 24-month-olds' ability to learn new information from language.

\section{Results and Discussion}

To be added after data collection and analyses are complete. 


\section{Visual Short-term Memory (VSTM) task: administered at 15 and 24 months}

\section{Stimuli}

In this adapted VSTM task, children view a screen with two sets of three rectangles of distinct colors - one on the left and one on the right side of the screen (see Figure 3).

\section{Procedure}

Both sets of triangles are shown for $500 \mathrm{~ms}$ with 250 -ms interval (when the screen is blank), for $20 \mathrm{~s}$ in total. During the 20 -s trial, one set of rectangles remains the same throughout the entire trial, while in the other set, one of the rectangles changes color every time the set 'blinks' (disappears for $250 \mathrm{~ms}$ and reappears on the screen). Each time, a different rectangle changes its color. No two rectangles within a set are of the same color at any given time. Each of the rectangles changes colors equal number of times through the duration of the trial. On average, children see nine different color combinations, each shown three times throughout the duration of the trial (see Figure 3). We present children with six 20-s trials. The side of the changing set of rectangles is counterbalanced among the trials. The order of the trials is randomized.

\section{Looking time data preparation}

Identical to the verbal refence task.

\section{Dependent variables}

Children looking preference for the changing set of rectangles will be measured:

$$
\mathrm{LT}_{\mathrm{vstm}}=\frac{\mathrm{LT}_{\text {change }}}{\mathrm{LT}_{\text {change }}+\mathrm{LT}_{\text {no change }}}
$$

\section{Preliminary analyses}


Preliminary analyses will test the effects of demographic factors and side of the target stimuli Analyses will be implemented by fitting Generalized Linear Models (GLM) using the glm function of the Stats package in R (R Core Team, 2013). If any such factor is significant in preliminary analyses, we will include it in main analyses.

\section{Analyses and Predictions}

In analyzing children's performance on the VSTM task, we expect to replicate the effects reported by Ross-sheehy et al. (2003) and observe a significant preference for the changing display. We will use an intercept-only GLM to evaluate this prediction using the $g l m$ function of the Stats package in $\mathrm{R}$ (with offset $=0.5$ to evaluate the intercept against the chance level).

\section{Results and Discussion}

To be added after data collection and analyses are complete.

\section{Spatial Cueing task: administered at 15 and 24 months}

\section{Stimuli}

In this task, adapted from Arredondo et al. (2022), children see a visual attention getter (a spinning rainbow wheel), a visual cue (an asterisk), and images of familiar toys (e.g., a ball, a colorful block, etc., see Figure 4a).

\section{Procedure}

In the beginning of each trial, children first see an attention getter in the center of the screen, displayed for $1000 \mathrm{~ms}$. Then, a visual cue - an asterisk - appears either on the left or the right side of the screen and remains displayed for $150 \mathrm{~ms}$, followed by a blank screen, which is displayed for $100 \mathrm{~ms}$. After that, children see a target image, which is displayed for $1500 \mathrm{~ms}$. In the Congruent condition, the image appears in former location of the asterisk. In the Incongruent 
condition, the image appears on the opposite side of the screen. Each child sees both conditions, presented in 12 blocks of 5 trials (60 trials in total), presented in a random order. Blocks are separated by a 10 -second resting period, during which an image of a dancing Elmo is presented in the center of the screen (see Figure 4).

\section{Looking time data preparation}

Identical to the verbal refence task.

\section{Dependent variables}

We will measure the average accuracy of children's looking following the cue and the latency of their gaze shift:

$$
\begin{aligned}
& \text { Accuracy }= \begin{cases}1, & \text { shifts gaze to the side of the target after seeing the cue } \\
0, & \text { shifts gaze to the opposite side, looks away, or no shift }\end{cases} \\
& M_{\text {acuracy_sc }}=\frac{N_{\text {accurate trials }}}{N_{\text {all trials }}} . \\
& \text { Latency }=\left\{\begin{array}{rlrl}
\text { time to firist gaze shift, } & & \text { Accuracy } & =1 \\
N A, & \text { Accuracy } & =0
\end{array}\right. \\
& M_{\text {latency }}=\frac{\text { Slatency }}{N_{\text {accurate trials }}} .
\end{aligned}
$$

In addition to these measures, which are based on Arredondo et al. (2022), we will calculate the number of trials $\left(\mathrm{NT}_{\text {change }}\right)$ it takes children to change their predictive looking strategy, measured by accuracy on each trial, after the type of block (congruent, incongruent) changes. This measure will serve as a proxy for executive control.

\section{Preliminary analyses}

Preliminary analyses will test the effects of demographic factors and side of the target stimuli. Analyses will be implemented by fitting Generalized Linear Models (GLM) using the 
glm function of the Stats package in R. If any such factor is significant in preliminary analyses, we will include it in main analyses.

\section{Analyses and Predictions}

In analyzing children's performance on the Spatial Cueing task, we expect to find that trial block (congruent, incongruent) will have significant effects on accuracy and latency of children's responses. We will use Tukey's HSD corrections to account for multiple comparisons. On incongruent trials, accuracy will be lower, and latency will be higher at both ages. We also expect that this effect will decrease with age, as children's command of attentional control becomes more robust. In addition, we predict that the number of trials it takes children to change their predictive looking strategy will decrease from 15 to 24 months.

\section{Results and Discussion}

To be added after data collection and analyses are complete.

\section{Relation between verbal reference and verbal testimony}

\section{Preliminary analyses}

Preliminary analyses will test the effects of demographic factors on children's performance on the VSTM and Spatial Cueing tasks (at each age: 15 and 24 months).

\section{Analyses and Predictions}

All analyses will be performed using Generalized Linear Mixed Models (GLMM) or Generalized Linear Models (GLM). GLMM will be used where estimated fixed effects (intercept, slope) can be affected by individual trials, which will be entered into the model as random effects. 
First, we will test the relation between children's performance on the verbal reference task at 15 months and their performance on the verbal testimony task at 24 months. This analysis will test our first hypothesis that verbal reference predicts learning from testimony.

Second, we will test our second hypothesis that differences in children's command of verbal reference may persist from 15 to 24 months, and may thus have concurrent as well as longitudinal effects on 24- month-olds' learning from verbal testimony. We will also conduct mediation analyses to assess whether a predictive relation between Verbal Reference and Verbal Testimony (both concurrent and longitudinal) is mediated by children's vocabulary.

Third, we test our third hypothesis that longitudinal and concurrent effects of verbal reference on learning from verbal testimony are distinct from more general advances in memory and attention. We will begin by evaluating the effects of VSTM and Spatial Cueing task on children's performance on verbal reference task and the verbal testimony task at each age. If either of these effects is significant, then to evaluate the unique contribution of verbal reference to verbal testimony, we will use two analytic strategies. First, we will add performance measures on VSTM and Spatial Cueing tasks to the models that evaluate the contribution of verbal reference to verbal testimony, both concurrently (all measures taken at 24 months) and longitudinally (verbal reference, VSTM, and Spatial Cueing measures taken at 15 months). If including VSTM and Spatial Cueing measures to the models changes the significance of the predictive relation between Verbal Reference and Verbal Testimony, we will conduct exploratory mediation analyses to see whether this relation is mediated by VSTM or Spatial Cueing. Second, we will residualize the dependent variables in both the verbal reference and the verbal testimony task on children's looking preference on their performance on the VSTM and Spatial Cueing tasks. Residuals will be calculated as the difference between the predicted values 
of each DV and their observed values. These residuals will be used to analyze the relation between children's performance on the verbal reference task and the verbal testimony task, both concurrently and longitudinally. That is, we will be evaluating the relation between children's performance on these tasks relative to their advances in vocabulary, attentional control, and memory at each age (15 and 24 months).

In analyzing the timecourse of children's net looking preference on the verbal testimony task, we will split the data into two groups based on children's overall net looking preference on the verbal reference task at 15 months $\left(\mathrm{LT}_{\mathrm{vr}-15 \mathrm{mo}}\right)$, using a median split, and analyze the divergences in between the conditions separately between the two groups. These analyses will be performed using cluster-based permutation analysis in 'eyetrackingr' package in R (Dink \& Ferguson, 2015). We expect that if children's performance on the verbal reference task at 15 months predicts their learning from testimony, then we will observe a larger divergence between the conditions in the group that exhibited larger net looking preference for the target at 15 months.

Finally, it is possible that we observe no significant effect of verbal reference on learning from verbal testimony, either longitudinally or concurrently. This outcome could be interpreted as evidence against the hypothesis that verbal reference is a prerequisite of children's ability to learn from testimony. But there is an alternative interpretation: that the current tasks are not sufficiently sensitive to reveal an effect of verbal reference on verbal testimony. To adjudicate between these interpretations, we will conder alternative designs in a follow-up investigation. If our analyses identify any significant relations between our verbal tasks with advances in memory, attentional control, and vocabulary, we will design follow-up studies zooming in on the nature of these relations. 


\section{Timeline}

We intend to begin data collection as soon as the current registered report is granted inprinciple acceptance. We expect that all data will be collected and analyzed within 18 months. If the Stage 1 review requests that the proposed experimental procedures be revised, we intend to accommodate this and to resubmit within one month of receiving reviewers' feedback.

\section{Apparatus}

For data collected in-lab, a Tobii T60XL corneal-reflection eyetracker will be used for stimulus presentation and data collection for all experimental tasks. The eyetracker has a sampling rate of $60 \mathrm{~Hz}$, and a display size of $57.3 \times 45 \mathrm{~cm}$.

\section{COVID-19 Contingency Plan}

The COVID-19 pandemic is currently preventing us from testing participants in the lab. All proposed experiments have been adapted to be conducted on Lookit - an online platform for developmental research (https://lookit.mit.edu; Scott \& Schulz, 2017). Using this platform, we will record children's eye movements using web-cameras (at an approximately $30 \mathrm{~Hz}$ sampling rate for most web-cameras) in their desktop or laptop computers while they view visual stimuli on their screen and listen to audio stimuli. Based on our recent experience conducting looking time studies on Lookit, we expect recruitment and retention rates similar to those we have historically experienced with in-lab experiments.

\section{Results}

To be added after data collection and analyses are complete.

\section{General Discussion}

To be added after data collection and analyses are complete. 


\section{References}

Akhtar, N. (2005). The robustness of learning through overhearing. Developmental Science, 8(2), 199-209. https://doi.org/10.1111/j.1467-7687.2005.00406.x

Anderson, D. R., \& Pempek, T. A. (2005). Television and Very Young Children. American Behavioral Scientist, 48(5), 505-522. https://doi.org/10.1177/0002764204271506

Arredondo, M. M., Aslin, R. N., Zhang, M., \& Werker, J. F. (2022). Attentional orienting abilities in bilinguals: Evidence from a large infant sample. Infant Behavior and Development, 66, 101683. https://doi.org/10.1016/j.infbeh.2021.101683

Arunachalam, S., \& Waxman, S. R. (2010). Meaning from syntax: Evidence from 2-year-olds. Cognition, 114(3), 442-446. https://doi.org/10.1016/j.cognition.2009.10.015

Astle, D. E., \& Scerif, G. (2011). Interactions between attention and visual short-term memory (VSTM): What can be learnt from individual and developmental differences? Neuropsychologia, 49(6), 1435-1445. https://doi.org/10.1016/j.neuropsychologia.2010.12.001

Bergelson, E. (2020). The Comprehension Boost in Early Word Learning: Older Infants Are Better Learners. Child Development Perspectives, 14(3), 142-149. https://doi.org/10.1111/cdep.12373

Bloom, L. (1993). The Transition from Infancy to Language: Acquiring the Power of Expression (1st ed.). Cambridge University Press. https://doi.org/10.1017/CBO9780511752797

Brooker, I., \& Poulin-Dubois, D. (2013). Is a Bird an Apple? The Effect of Speaker Labeling Accuracy on Infants' Word Learning, Imitation, and Helping Behaviors. Infancy, 18, E46-E68. https://doi.org/10.1111/infa.12027 
Cheng, C., Kaldy, Z., \& Blaser, E. (2019). Focused attention predicts visual working memory performance in 13-month-old infants: A pupillometric study. Developmental Cognitive Neuroscience, 36, 100616. https://doi.org/10.1016/j.den.2019.100616

Clark, H. H., \& Bangerter, A. (2004). Changing Ideas about Reference. In I. A. Noveck \& D. Sperber (Eds.), Experimental Pragmatics (pp. 25-49). Palgrave Macmillan UK. https://doi.org/10.1057/9780230524125_2

Cohen, J. (2013). Statistical Power Analysis for the Behavioral Sciences (2nd ed.). Routledge. https://doi.org/10.4324/9780203771587

Deacon, T. (1997). The symbolic species. New York: W.

Dink, J. W., \& Ferguson, B. (2015). EyetrackingR: An R Library for Eye-tracking Data Analysis. http://www.eyetrackingr.com

Faul, F., Erdfelder, E., Lang, A.-G., \& Buchner, A. (2007). G*Power 3: A flexible statistical power analysis program for the social, behavioral, and biomedical sciences. Behavior Research Methods, 39(2), 175-191. https://doi.org/10.3758/BF03193146

Fenson, L., Pethick, S., Renda, C., Cox, J. L., Dale, P. S., \& Reznick, J. S. (2000). Short-form versions of the MacArthur Communicative Development Inventories. Applied Psycholinguistics, 21(1), 95-116. https://doi.org/10.1017/S0142716400001053

Ferguson, B., Graf, E., \& Waxman, S. R. (2018). When Veps Cry: Two-Year-Olds Efficiently Learn Novel Words from Linguistic Contexts Alone. Language Learning and Development, 14(1), 1-12. https://doi.org/10.1080/15475441.2017.1311260

Floor, P., \& Akhtar, N. (2006). Can 18-Month-Old Infants Learn Words by Listening In on Conversations? Infancy, 9(3), 327-339. https://doi.org/10.1207/s15327078in0903_4 
Forbes, S. H., \& Plunkett, K. (2019). Infants show early comprehension of basic color words. Developmental Psychology, 55(2), 240-249. https://doi.org/10.1037/dev0000609

Foushee, R., Srinivasan, M., \& Xu, F. (2021). Self-directed learning by preschoolers in a naturalistic overhearing context. Cognition, 206, 104415. https://doi.org/10.1016/j.cognition.2020.104415

Frank, M. C., Braginsky, M., Yurovsky, D., \& Marchman, V. A. (2017). Wordbank: An open repository for developmental vocabulary data. Journal of Child Language, 44(3), 677694. https://doi.org/10.1017/S0305000916000209

Galazka, M. A., \& Ganea, P. A. (2014). The role of representational strength in verbal updating: Evidence from 19- and 24-month-olds. Journal of Experimental Child Psychology, 121, 156-168. https://doi.org/10.1016/j.jecp.2013.12.002

Gallerani, C. M., Saylor, M. M., \& Adwar, S. (2009). Mother-Infant Conversation About Absent Things. Language Learning and Development, 5(4), 282-293. https://doi.org/10.1080/15475440902897604

Ganea, P. A., Fitch, A., Harris, P. L., \& Kaldy, Z. (2016). Sixteen-month-olds can use language to update their expectations about the visual world. Journal of Experimental Child Psychology, 151, 65-76. https://doi.org/10.1016/j.jecp.2015.12.005

Ganea, P. A., \& Harris, P. L. (2010). Not Doing What You Are Told: Early Perseverative Errors in Updating Mental Representations via Language. Child Development, 81(2), 457-463. https://doi.org/10.1111/j.1467-8624.2009.01406.x

Ganea, P. A., \& Harris, P. L. (2013). Early limits on the verbal updating of an object's location. Journal of Experimental Child Psychology, 114(1), 89-101. https://doi.org/10.1016/j.jecp.2012.04.013 
Ganea, P. A., \& Saylor, M. M. (2013). Representational Constraints on Language Development: Thinking and Learning About Absent Things. Child Development Perspectives, 7(4), 227-231. https://doi.org/10.1111/cdep.12045

Ganea, P. A., Shutts, K., Spelke, E. S., \& DeLoache, J. S. (2007). Thinking of Things Unseen: Infants' Use of Language to Update Mental Representations. Psychological Science, 18(8), 734-739.

Harris, P. L., \& Corriveau, K. H. (2011). Young children's selective trust in informants. Philosophical Transactions of the Royal Society B: Biological Sciences, 366(1567), 1179-1187. https://doi.org/10.1098/rstb.2010.0321

Harris, P. L., \& Lane, J. D. (2014). Infants Understand How Testimony Works. Topoi, 33(2), 443-458. https://doi.org/10.1007/s11245-013-9180-0

Hendrickson, K., \& Sundara, M. (2017). Fourteen-month-olds' decontextualized understanding of words for absent objects. Journal of Child Language, 44(1), 239-254. https://doi.org/10.1017/S0305000915000756

Koenig, M. A., \& Harris, P. L. (2005). The role of social cognition in early trust. Trends in Cognitive Sciences, 9(10), 457-459. https://doi.org/10.1016/j.tics.2005.08.006

Locke, J. (1847). An Essay Concerning Human Understanding. Kay \& Troutman.

Luchkina, E., Sobel, D. M., \& Morgan, J. L. (2018). Eighteen-month-olds selectively generalize words from accurate speakers to novel contexts. Developmental Science, 21(6), e12663. https://doi.org/10.1111/desc.12663

Luchkina, E., \& Waxman, S. (2022). Semantic priming supports infants' ability to represent and name unseen objects. Proceedings of the 44th Annual Meeting of the Cognitive Science 
Society. Annual Meeting of the Cognitive Science Society, Toronto, Canada. https://doi.org/10.31219/osf.io/tp39b

Luchkina, E., \& Waxman, S. R. (2021a). Acquiring verbal reference: The interplay of cognitive, linguistic, and general learning capacities. Infant Behavior and Development.

Luchkina, E., \& Waxman, S. R. (2021b). Semantic priming supports infants' ability to learn names of unseen objects. PLOS ONE, 16(1), e0244968. https://doi.org/10.1371/journal.pone.0244968

Luchkina, E., \& Xu, F. (2021). From social contingency to verbal reference: A constructivist hypothesis. Psychological Review. 10.31219/osf.io/eyx2b

Luchkina, E., Xu, F., Sobel, D., \& Morgan, J. (2020). Sixteen-month-olds comprehend unanchored absent reference. Proceedings of the 42nd Annual Meeting of the Cognitive Science Society, 1399-1404. https://doi.org/10.31219/osf.io/5tc6d

Miller, G. A. (1990). The Place of Language in a Scientific Psychology. Psychological Science, 1(1), 7-14. https://doi.org/10.1111/j.1467-9280.1990.tb00059.x

O’Doherty, K., Troseth, G. L., Shimpi, P. M., Goldenberg, E., Akhtar, N., \& Saylor, M. M. (2011). Third-Party Social Interaction and Word Learning From Video: Social Interaction and Video. Child Development, 82(3), 902-915. https://doi.org/10.1111/j.14678624.2011.01579.x

Osina, M. A., Saylor, M. M., \& Ganea, P. A. (2013). When familiar is not better: 12-month-old infants respond to talk about absent objects. Developmental Psychology, 49(1), 138-145. https://doi.org/10.1037/a0027903 
Osina, M. A., Saylor, M. M., \& Ganea, P. A. (2014). Object Locations, Identity and Absent Reference Understanding at 12 Months. Infancy, 19(1), 65-81. https://doi.org/10.1111/infa.12031

Özdemir, B., \& Ganea, P. A. (2020). Variability in toddlers' ability to verbally update their mental representations of absent objects. Journal of Experimental Child Psychology, 196, 104843. https://doi.org/10.1016/j.jecp.2020.104843

Pruden, S. M., Hirsh-Pasek, K., Golinkoff, R. M., \& Hennon, E. A. (2006). The Birth of Words: Ten-Month-Olds Learn Words Through Perceptual Salience. Child Development, 77(2), 266-280. https://doi.org/10.1111/j.1467-8624.2006.00869.x

Robb, M. B., Richert, R. A., \& Wartella, E. A. (2009). Just a talking book? Word learning from watching baby videos. British Journal of Developmental Psychology, 27(1), 27-45. https://doi.org/10.1348/026151008X320156

Ross-sheehy, S., Oakes, L. M., \& Luck, S. J. (2003). The Development of Visual Short-Term Memory Capacity in Infants. Child Development, 74(6), 1807-1822. https://doi.org/10.1046/j.1467-8624.2003.00639.x

Saylor, M. M. (2004). Twelve- and 16-month-old infants recognize properties of mentioned absent things. Developmental Science, 7(5), 599-611. https://doi.org/10.1111/j.14677687.2004.00383.x

Saylor, M. M., Osina, M., Tassin, T., Rose, R., \& Ganea, P. (2016). Creature feature: Preschoolers use verbal descriptions to identify referents. Journal of Experimental Child Psychology, 152, 205-220. https://doi.org/10.1016/j.jecp.2016.07.005

Scolnikov, S. (2006). Language and dialogue in Plato. Language and Dialogue in Plato. Classica-Revista Brasileira de Estudos Clássicos, 19(2), 180-186. 
Sobel, D. M., \& Kushnir, T. (2013). Knowledge matters: How children evaluate the reliability of testimony as a process of rational inference. Psychological Review, 120(4), 779-797. https://doi.org/10.1037/a0034191

Team, R. C. (2013). R: A language and environment for statistical computing. http://r.meteo.uni.wroc.pl/web/packages/dplR/vignettes/intro-dplR.pdf

Waxman, S. R., \& Gelman, S. A. (2009). Early word-learning entails reference, not merely associations. Trends in Cognitive Sciences, 13(6), 258-263.

https://doi.org/10.1016/j.tics.2009.03.006

Waxman, S. R., \& Lidz, J. L. (2007). Early World Learning. In W. Damon \& R. M. Lerner (Eds.), Handbook of Child Psychology (p. chpsy0207). John Wiley \& Sons, Inc. https://doi.org/10.1002/9780470147658.chpsy0207 


\section{Figures}

\begin{tabular}{|c|c|c|c|c|c|c|c|}
\hline \multirow{2}{*}{\multicolumn{5}{|c|}{ PRIMING (35 S) }} & \multicolumn{3}{|c|}{ TEST (10 s) } \\
\hline & & & & & $2 s$ & $2 \mathrm{~s}$ & $6 s$ \\
\hline Auditory stream: & & & & & & & \\
\hline $\begin{array}{l}\text {...Look, an } \\
\text { apple!.. }\end{array}$ & $\begin{array}{l}\text {...Look, an } \\
\text { orange!.. }\end{array}$ & $\begin{array}{l}\text {...Look, a } \\
\text { banana!.. }\end{array}$ & $\begin{array}{l}\text {...Look, } \\
\text { modi!.. }\end{array}$ & $\begin{array}{l}\text {... Let's find } \\
\text { the modi!.. }\end{array}$ & & $\begin{array}{l}\ldots \text { Where is } \\
\text { the modi?.. }\end{array}$ & $\begin{array}{l}{[2 \mathrm{~s} \text { delay }] \text { Can you }} \\
\text { find the modi? }\end{array}$ \\
\hline
\end{tabular}

Figure 1a. Verbal reference task. A representative set of visual and auditory stimuli. At Test, children are asked to identify the referent of the novel word from Priming (e.g., modi).

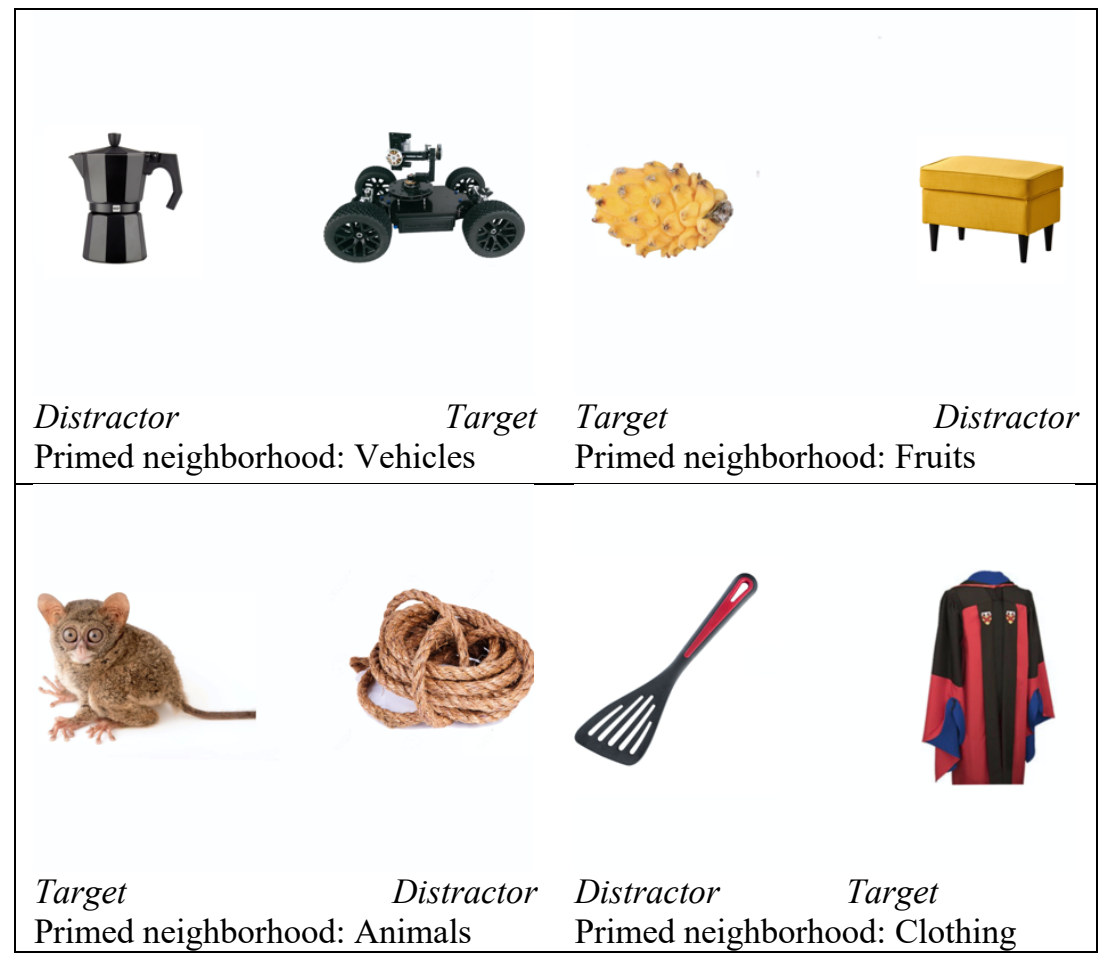

Figure 1b. Verbal reference task. Test stimuli on each of four trials: (1) a drone car and a coffee maker, (2) a pitaya fruit and an ottoman, (3) a sugar glider and a rope, and (4) a graduation gown and a spatula. Images of objects in all pairs were chosen to have a similar color or color combination and similar luminance. 


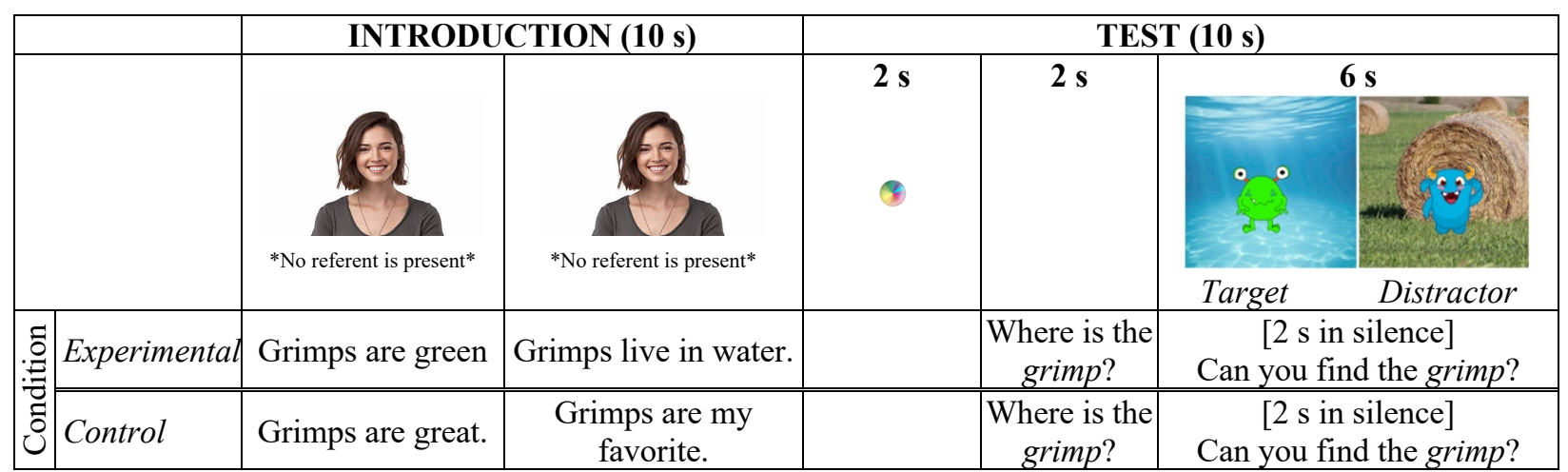

Figure 2a. Verbal testimony task. A representative set of stimuli in the Experimental and Control conditions (betweensubjects). Each child participates in 4 trials. In the Experimental condition, the actor names a novel creature, describing its color and habitat. In the Control condition, the actor names a novel creature but provides no information about its color or habitat. At Test, children are asked to look to the referent of the name. Children in the Experimental condition should prefer looking to the target object; those in the Control condition should perform at chance.

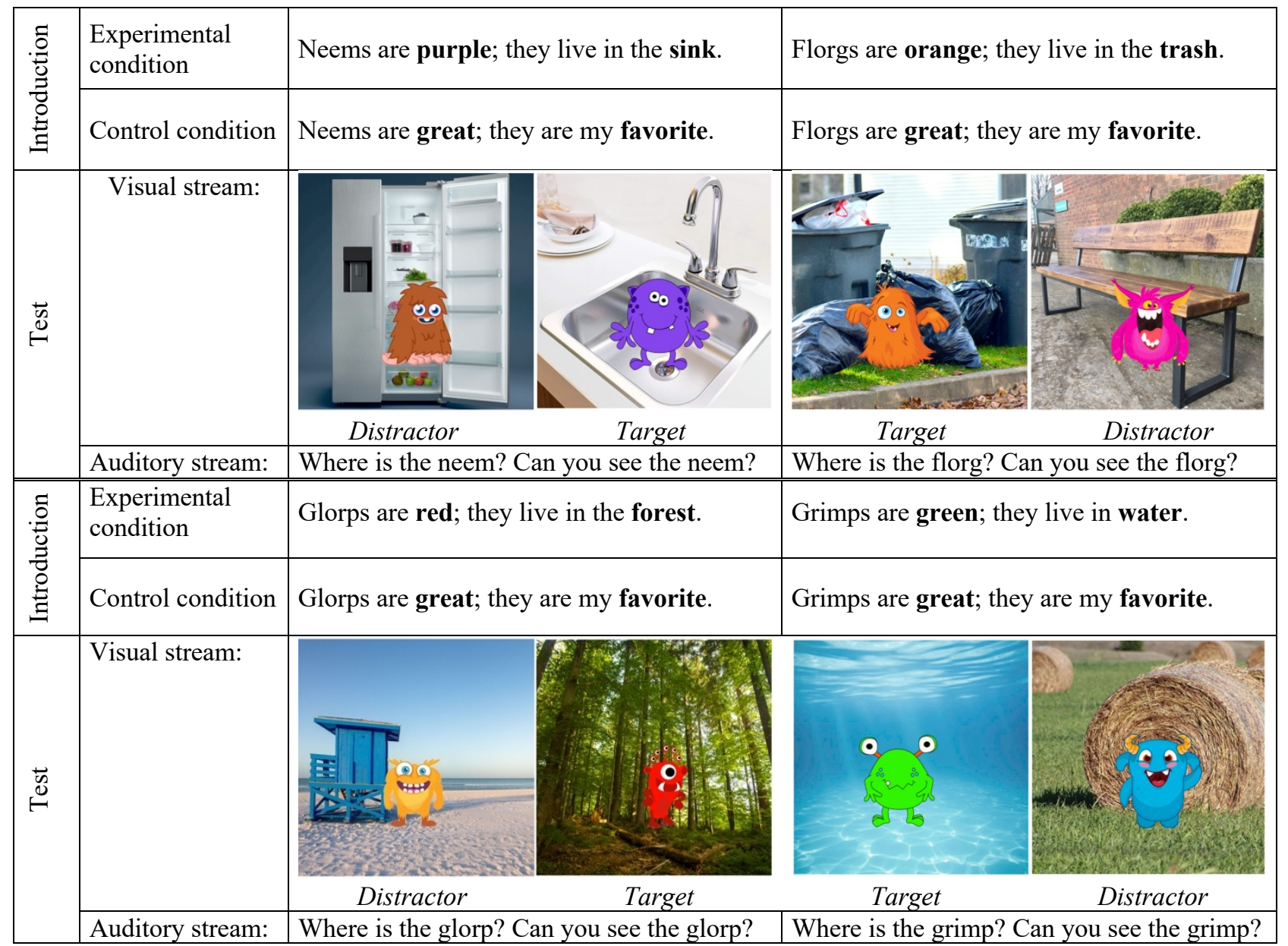

Figure 2b. Verbal testimony task. Verbal decrepitations provided during Introduction and Test stimuli for each trial in each condition. On two trials, the creatures' habitat is nature, on the other two trials the habitat is human residence. 


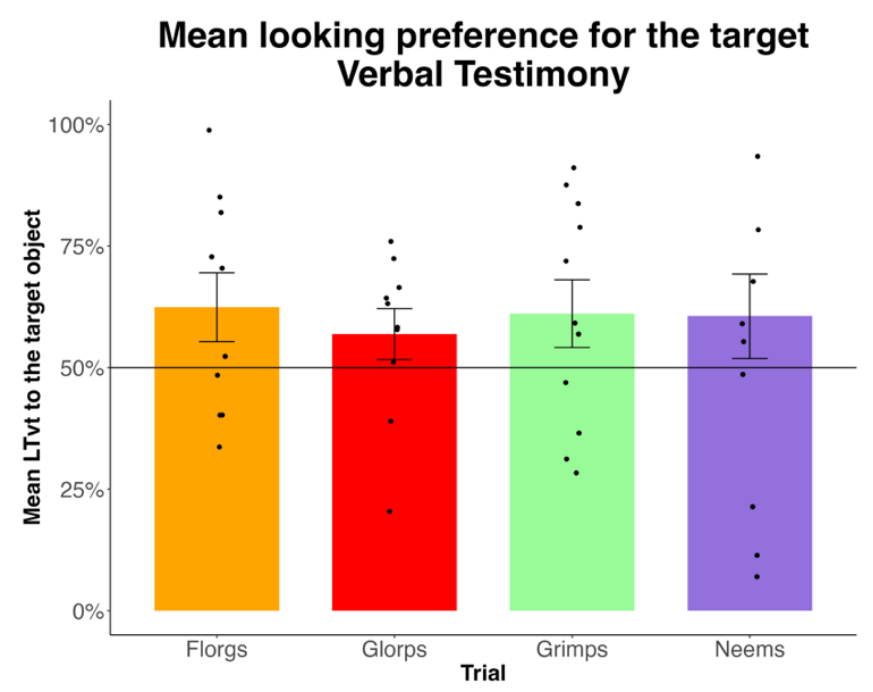

Figure 2c. Verbal testimony task. Pilot results $(N=13)$. Error bars represent standard error, black dots represent individual data points.

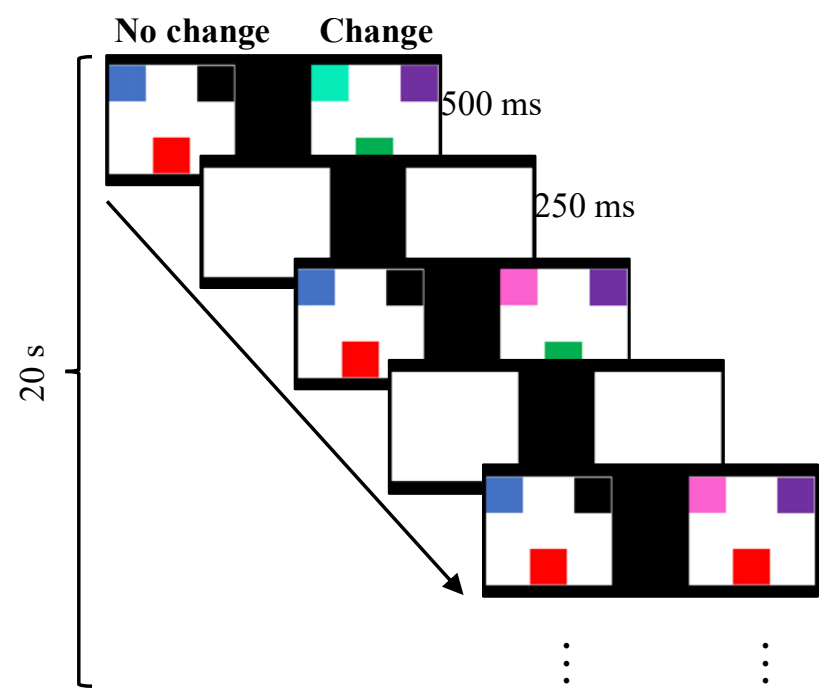

Figure 3. Visual Short-Term Memory task. A representative set of visual stimuli on the VSTM task.

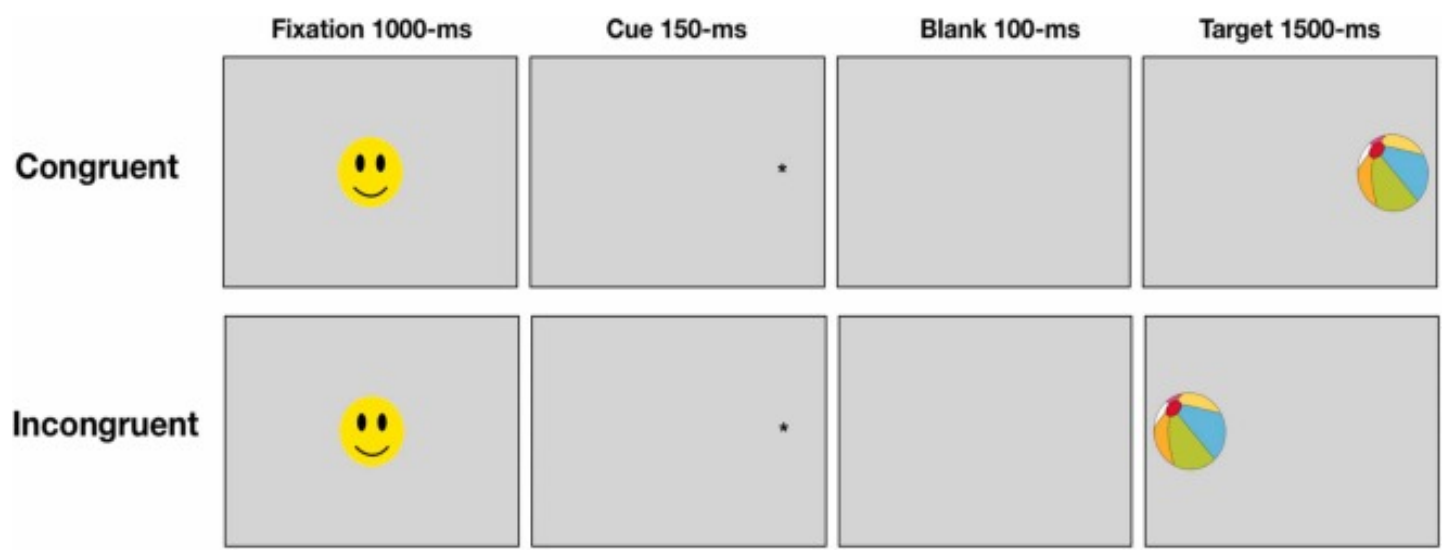

Figure 4. Spatial Cueing Task. A representative set of test trials. Reprinted from Arredondo et al. (2022), Infant Behavior and Development. 
${ }^{1}$ While vocabulary begins to develop the first year of life (see Bergelson, 2020, for a recent review) these children likely do not possess a referential link between words and mental representations. Indeed, empirical evidence suggests that children's word knowledge in the first year may be based on perceptual associations rather than referential links between words, referents, and their mental representations (e.g., Pruden et al., 2006). Our definition of a referential link entails that representations are not simply accessed via words (as a representation of a music instrument can be accessed via its sound). It requires an awareness that words are used intentionally by people for communication purposes and can be used to communicate about perceptually unavailable entities. This awareness enables one to not only access existing representations but form new representations based on verbal information. ${ }^{2}$ These children, however, have more difficulty updating their knowledge of location of a hidden object than of its property (Özdemir \& Ganea, 2020). This may be related to limitations in working memory (Ganea \& Harris, 2013). ${ }^{3}$ The original task (Luchkina \& Waxman, 2021b) included two control conditions designed to assess the possibility that children's test performance in the Semantic Priming condition (1) was driven by their intrinsic preferences for particular images and (2) by a preference to look to a semantically related object during Test, without linking the representation of it to the novel word. Because both possibilities were ruled out (see Luchkina \& Waxman, under review), we do not include these control conditions here. We do still account for children's potential bias in looking by subtracting their looking preference for the target during the silent presentation of the stimuli for the first $2 \mathrm{~s}$. ${ }^{4}$ We chose to calculate the net looking preference instead of the gross looking preference over duration of the trial $(0-6000 \mathrm{~ms})$ due to a potential familiarity effect of the target object category. Infants in Luchkina and Waxman (2022) showed a sharp increase in looking to the target object within $100 \mathrm{~ms}$ of the image display onset, which could not have been driven by the target word or the semantic neighborhood priming - the effect was present in all conditions. Infants' looking preference reverted to the chance level by $2000 \mathrm{~ms}$ from the target image onset in all conditions. All subsequent increases in looking to the target in the Semantic Priming condition were driven by hearing the target word. This the initial sharp increase in looking to the target object was likely driven by the overall higher familiarity of the target object category membership (e.g., fruits) than the distractor object (e.g., furniture). ${ }^{5}$ The index "vr" stands for "verbal reference"; it is used to distinguish the net looking preference on the Verbal Reference task from the net looking preference on the Verbal Testimony task, indexed as "vt".

${ }^{6}$ The same object is considered "target" in each condition (even if the information presented during Introduction does not make it a likely choice; this simplifies our comparison of LT across conditions). 